Claremont Colleges

Scholarship@ Claremont

All HMC Faculty Publications and Research

HMC Faculty Scholarship

8-1-2001

\title{
An Inverse Function Theorem via Continuous Newton's Method
}

\author{
Alfonso Castro \\ Harvey Mudd College \\ J.W. Neuberger \\ University of North Texas
}

\section{Recommended Citation}

Castro, Alfonso and J. W. Neuberger. “An inverse function theorem via continuous Newton's method”, Nonlinear Analysis 47 (2001), pp. 3223-3229.

This Article - postprint is brought to you for free and open access by the HMC Faculty Scholarship at Scholarship @ Claremont. It has been accepted for inclusion in All HMC Faculty Publications and Research by an authorized administrator of Scholarship @ Claremont. For more information, please contact scholarship@cuc.claremont.edu. 


\title{
An Inverse Function Theorem via Continuous Newton's Method
}

\author{
Alfonso Castro and J.W. Neuberger \\ Dept. of Mathematics, University of Texas, San Antonio, TX 78249 \\ Dept. of Mathematics, University of North Texas, Denton, TX 76203
}

\begin{abstract}
We prove an inverse function theorem of the Nash-Moser type. The main difference between our method and that of $[4]$ is that we use continuous steepest descent while [4] uses a combination of Newton type iterations and approximate inverses. We bypass the loss of derivatives problem by working on finite dimensional subspaces of infinitely differentiable functions.
\end{abstract}

Key words: Inverse Function Theorem Continuous Newton's Method

\section{Introduction}

Inverse function theorems have long had a prominent role in analysis, particularly in the study of differential equations. In [4] there is an inverse function theorem whose proof uses a form of conventional Newton's method. In the present note we use a version of continuous Newton's method to give a new inverse function theorem. Our hypothesis is suggested from [4] but a direct comparison has not yet been established. We believe, however, that the present result covers a substantial portion of the cases covered by the hypothesis in [4].

Suppose each of $H$ and $K$ is a Banach space, $r>0$ and $F$ is a $C^{1}$ function from the open ball $B_{r}(0)$ in $H$, centered at 0 , so that $F(0)=0$. We intend to give a condition on a member $g$ of $K$ so that there exists $\gamma>0$ such that $\operatorname{tg} \in R(F)$ if $0 \leq t \leq \gamma$. In intended applications, $H$ may be the Sobolev space $H^{1,2}(\Omega)$ for some region $\Omega$ in a Euclidean space and $K$ may be $L_{2}(\Omega)$. The function $F$ then may be a nonlinear differential operator. 
For a motivating example we essentially follow [4] by choosing $F$ defined by

$$
F(u)=u_{1} \text { for } u \in H^{1,2}(\Omega)
$$

where here $\Omega=[0,1]^{2}$ and the subscript on $u$ indicates partial differentiation in the first argument of $u$. Some reflection yields that the range of $F$ can not be all of $L_{2}(\Omega)$ since many members $g$ of that space lack sufficient smoothness to be in that range. For some members $g$ of $L_{2}(\Omega)$, namely those members of $L_{2}(\Omega)$ which are also in $H^{1,2}(\Omega)$, there is a solution $u$ to

$$
F(u)=g
$$

which is in $H^{1,2}(\Omega)$. To be more specific, suppose $r>0$ and $H^{\prime}$ is a subset of $H^{1,2}(\Omega)$, uniformly bounded in the norm of that space. Observe that there is $\gamma>0$ so that if $0 \leq t \leq \gamma$ and $g \in H^{\prime}$, then there is

$$
u \in H^{1,2}(\Omega) \text { with }\|u\|_{H^{1,2}(\Omega)} \leq r
$$

so that

$$
F(u)=t g
$$

\section{An Inverse Function Theorem}

Return to the general setting of the introduction, that is suppose $r>0$,

$$
F: B_{r}(0) \subset H \rightarrow K,
$$

$F$ is so that $F(0)=0$ and $F$ is $C^{1}$.

Theorem 1 Suppose $g \in K$ and there is a function $h$ with domain $B_{r}(0) \subset H$ which is Lipschitz continuous so that

$$
F^{\prime}(x) h(x)=g \text { for }\|x\|_{H} \leq r .
$$

Then there is $\gamma>0$ so that if $0 \leq t \leq \gamma$, there is $u \in B_{r}(0)$ so that

$$
F(u)=t g
$$

Proof: Under the hypothesis of the theorem, denote by $\gamma$ a positive number so that there is a unique solution $z$ on $[0, \gamma]$ to

$$
z(0)=0 \text { and } z^{\prime}(t)=h(z(t)) \text { with }\|z(t)\| \leq r \text { for } t \in[0, \gamma]
$$


(that there is such a number $\gamma$ follows from the basic existence and uniqueness theorem for ordinary differential equations). Then

$$
F^{\prime}(z(t)) z^{\prime}(t)=F^{\prime}(z(t))(h(z(t))=g
$$

i.e.,

$$
(F(z))^{\prime}(t)=g \text { for } t \in[0, \gamma]
$$

Hence,

$$
F(z(t))=t g \text { for } \in[0, \gamma],
$$

and the argument is complete.

Note that we have not required uniqueness of solution $k \in H$ to

$$
F^{\prime}(x) k=g
$$

for any $x \in B_{r}(0)$, but rather that solutions to 3 for various $x \in B_{r}(0)$ can be fit together in a smooth enough way in order to provide a function $h$ satisfying the hypothesis of the theorem.

\section{Discussion}

Here we attempt to justify calling the above process a version of continuous Newton's method. Suppose here that $F$ is a function from $H$ to $K$ so that $F(0)=0$ and $\left(F^{\prime}(x)\right)^{-1}$ exists and is in $L(K, H)$ for each $x \in H$. Suppose furthermore that $\left(F^{\prime}(\cdot)\right)^{-1}$ is locally Lipschitz. Given $g \in K$, conventional continuous Newton's method for finding $u \in H$ such that $F(u)=g$ might consist of first finding $z:[0, \infty) \rightarrow H$ so that

$$
z(0)=0 \text { and } z^{\prime}(t)=-\left(F^{\prime}(z(t))\right)^{-1}(F(z(t))-g) \text { for } t \geq 0
$$

and then seeking

$$
u=\lim _{t \rightarrow \infty} z(t)
$$

so that $F(u)=g$.

Assuming we have $z$ satisfying (4), we then have

$$
F^{\prime}(z(t)) z^{\prime}(t)=-(F(z(t))-g) \text { for } t \geq 0,
$$

and consequently

$$
(F(z)-g)^{\prime}(t)=-(F(z(t))-g) \text { for } t \geq 0 .
$$


Hence we have

$$
F(z(t))-g=e^{-t}(F(z(0))-g) \text { for } t \geq 0 .
$$

Using 5 and the fact that $F(z(0))=0$ we substitute in 4 to obtain an alternate expression

$$
z(0)=0, z^{\prime}(t)=e^{-t}\left(F^{\prime}(z(t))^{-1} g \text { for } t \geq 0 .\right.
$$

Deleting the exponential factor in the above just changes the time scale from $[0, \infty)$ to $[0,1)$, which leaves us with

$$
z(0)=0, z^{\prime}(t)=\left(F^{\prime}(z(t))\right)^{-1} g \text { for } t \in[0,1) .
$$

This provided motivation for the process 2 in which it is not assumed that $\left(F^{\prime}(x)\right)^{-1}$ for $x \in B_{r}(0)$, exist, but rather that for a fixed $g \in K$ and any $x \in B_{r}(0)$, there is $k \in H$ such that

$$
F^{\prime}(x) k=g
$$

(and these solutions $k$ for $x \in B_{r}(0)$ can be fit together to make a function $h$ as in the hypothesis of the theorem).

\section{Application: range of the sum of two maximal monotone opera- tors}

Finally we consider the semilinear boundary value problem

$$
-\Delta u+f(u)=g \text { in } \Omega, u(x)=0 \text { for } x \in \partial \Omega,
$$

where $\Omega$ is a smooth bounded region in $R^{n}$ with $n>2$, and $f: R \rightarrow R$ is a monotonically increasing function with supercritical growth (i.e.,

$$
\liminf _{|t| \rightarrow \infty} f(t) / t^{\rho}>0
$$

for some $\rho>(n+2) /(n-2))$ and has a locally Lipschitzian derivative. For the sake of simplicity in the calculations we assume that $f(0)=0$. Due to the growth of $f$, the sum of the maximal monotone operators defined by $-\Delta$ and $f$ is not maximal monotone (see [1]) and general theory does not provide adequate information on its range. However letting $p>\min \{1, n / 2\}$ and taking $H$ to be the Sobolev space $H_{0}^{2, p}(\Omega)$ of functions having second order derivatives in $L^{p}(\Omega)$ and vanishing on the boundary of $\Omega$, and $K$ the space $L^{p}(\Omega)$ we see that for each $u \in H, g \in K$ the equation

$$
-\Delta v+f^{\prime}(u) v=g \text { in } \Omega, u(x)=0 \text { for } x \in \partial \Omega,
$$


has a unique solution $v=h(u)$ (see Thoerem 9.15 of [3]). In order to apply Theorem 1 we prove the following result.

Lemma 1 The function $h: H \rightarrow H$ given by $h(u)=v$, where $u$, $v$ are as in (7), is bounded on bounded sets and locally lipzchitzian.

Proof: Let $B$ be a bounded subset of $H$. By the Sobolev imbedding theorem (see Corollary 7.11 of [3]), without loss of generality we may assume that $\|u\|_{\infty} \leq 1$ for $u \in B$. Let $t>((n-2) p-n) / n$ be an odd positive integer. Multiplying (7) by $v^{t}$, and using that $f^{\prime} \geq 0$ and Holder's inequality we have

$$
\begin{aligned}
\int_{\Omega} \nabla v^{(t+1) / 2} \cdot \nabla v^{(t+1) / 2} d x & \leq \frac{(t+1)^{2}}{4 t} \int_{\Omega} g v^{t} d x \\
& \leq \frac{(t+1)^{2}}{4 t}\|g\|_{s}\|v\|_{(t+1) n /(n-2)}^{t}
\end{aligned}
$$

where $s=(t+1) n /(n+2 t) \leq n / 2$. Let $C>0$ (see again Corollary 7.11 of [3]) be a constant such that

$$
\left(\int_{\Omega}|w|^{2 n /(n-2)} d x\right)^{(n-2) /(2 n)} \leq C\left(\int_{\Omega} \nabla w \cdot \nabla w d x\right)^{1 / 2},
$$

for all $w \in H_{0}^{1,2}$. Taking $w=v^{(t+1) / 2}$ and using (8) we see that

$$
\|v\|_{n(t+1) /(n-2)} \leq \frac{C^{2}(t+1)^{2}}{4 t}\|g\|_{s}
$$

Since $s \leq n / 2<p$, we see that there exists a constant $M$ such that

$$
\|v\|_{p} \leq M\|g\|_{n / 2}
$$

Thus $\left\|g-f^{\prime}(u) v\right\|_{p} \leq\|g\|_{p}+M_{1}\|v\|_{p}$, where $M_{1}=$ $\leq\|u\|_{\infty}+$ 1\}. Therefore by a priori estimes for elliptic bounaary value problems (see Theorem 9.15 of [3]) we infer $\|v\|_{H} \leq M_{2}$, with $M_{2}$ depending on $M_{1}, \Omega$, and $p$. Thus $h$ is bounded in bounded sets.

Let $u_{1}, u_{2}, v_{1}, v_{2} \in H$ be such that

$$
-\Delta v_{i}+f^{\prime}\left(u_{i}\right) v_{i}=g
$$

for $i=1,2$, with $\left\|u_{1}-u_{2}\right\|_{H} \leq 1$. An elementary algebraic manipulation shows that $-\Delta\left(v_{1}-v_{2}\right)+f^{\prime}\left(u_{1}\right)\left(v_{1}-v_{2}\right)=\left(f^{\prime}\left(u_{2}\right)-f^{\prime}\left(u_{1}\right)\right) v_{2}$. From Lemma 9.17 
of [3], there exists a positive constant $C_{q}$ for each $q \in(1, \infty)$ such that if

$$
-\Delta w+f^{\prime}\left(u_{1}\right) w=y
$$

with $y \in L^{q}(\Omega)$ then $w \in H_{0}^{2, q}(\Omega)$ and

$$
\|w\|_{H_{0}^{2, q}(\Omega)} \leq C_{q}\|y\|_{q}
$$

Also by the Sobolev imbedding theorem there exists a constant $\bar{C}$ such that

$$
\|z\|_{\infty} \leq \bar{C}\|z\|_{H}
$$

for all $z \in H$. Therefore

$$
\begin{aligned}
\left\|v_{1}-v_{2}\right\|_{H} & \leq C_{p}\left\|\left(f^{\prime}\left(u_{1}\right)-f^{\prime}\left(u_{2}\right)\right) v_{2}\right\|_{K} \leq A C_{p}\left\|u_{1}-u_{2}\right\|_{\infty}\left\|v_{2}\right\|_{K} \\
& \leq A C_{p} M_{2}\left\|u_{1}-u_{2}\right\|_{\infty} \leq A C_{p} M_{2} \bar{C}\left\|u_{1}-u_{2}\right\|_{H}
\end{aligned}
$$

where $A$ is a Lipschitz constant for $f^{\prime}$ on $\left[-\bar{C}\left(\|u\|_{\infty}+1\right), \bar{C}\left(\|u\|_{\infty}+1\right)\right]$. This proves that $h$ is a locally Lipchitzian function, which proves the lemma.

Now combining Lemma 1 and Theorem 1 we prove that for any $g \in K$ the equation (6) has a solution.

Theorem 2 Under the above assumptions, for each $g \in K$ the equation (6) has a solution.

Proof: Let $F: H \rightarrow K$ be the operator defined by $F(u)=-\Delta u+f \circ u$. Let $z(t)$ be as in (2). Multiplying the equation $F(z(t))=t g$ by $|f(z(t))|^{p-2} f(z(t))$ we see that

$$
\int_{\Omega}|f(z(t))|^{p} d x \leq t \int_{\Omega}|g||f(z(t))|^{p-1} d x .
$$

Hence, by Holder's inequality, we see that $\|f(z(t))\|_{K}$ is bounded on bounded intervals of $[0, \infty)$. Thus, by Theorem 9.15 of $[3],\|z(t)\|_{H}$ is bounded on bounded intervals. Since $h$ is defined on all of $H$ it follows that $z$ is defined on $[0, \infty)$. In particular $F(z(1))=g$, which proves the theorem.

\section{References}

[1] H. Brezis, Opératerus Maximaux Monotones et Semi-groupes de Contractions dan les Espaces de Hilbert, North Holland, Mathematics Studies No. 5 (1973). 
[2] A. Castro and J. W. Neuberger, An Inverse Function Theorem, Contemporary Mathematics, Vol. 221 (1999), 127-132.

[3] D. Gilbarg and N. Trudinger, Elliptic Partial Differential Equations of Second Order, Berlin, New York, Springer-Verlag (1983).

[4] J. Moser, A Rapidly Convergent Iteration Methad and Non-Linear Differential Equations, Ann. Scuola Normal Sup. Pisa, 20 (1966), 265-315. 